\title{
Omega-3 fatty acids: potential role in the management of early Alzheimer's disease
}

This article was published in the following Dove Press journal:

Clinical Interventions in Aging

24 March 2010

Number of times this article has been viewed

\author{
Gregory A Jicha \\ William R Markesbery \\ University of Kentucky \\ Alzheimer's Disease Center and \\ the Sanders-Brown Center on Aging \\ University of Kentucky College of \\ Medicine, Lexington, KY, US
}

\begin{abstract}
Omega-3 fatty acids are essential for brain growth and development. They play an important role throughout life, as critical modulators of neuronal function and regulation of oxidative stress mechanisms, in brain health and disease. Docosahexanoic acid (DHA), the major omega-3 fatty acid found in neurons, has taken on a central role as a target for therapeutic intervention in Alzheimer's disease (AD). A plethora of in vitro, animal model, and human data, gathered over the past decade, highlight the important role DHA may play in the development of a variety of neurological and psychiatric disorders, including AD. Cross sectional and prospective cohort data have demonstrated that reduced dietary intake or low brain levels of DHA are associated with accelerated cognitive decline or the development of incipient dementia, including AD. Several clinical trials investigating the effects of omega-3 fatty acid supplementation in AD have been completed and all failed to demonstrate its efficacy in the treatment of $\mathrm{AD}$. However, these trials produced intriguing data suggesting that the beneficial effects of omega- 3 fatty acid supplementation may depend on the stage of disease, other dietary mediators, and apolipoprotein E status.
\end{abstract}

Keywords: Alzheimer's disease, omega-3 fatty acids, oxidative stress, clinical studies, treatment

\section{Introduction}

Alzheimer' disease (AD) is a progressive neurodegenerative disorder that represents the major cause of dementia in the world today. ${ }^{1}$ Over 5 million persons in the US currently suffer from this fatal disease and that number is expected to quadruple by the year 2050, unless prevention efforts or disease modifying therapies are developed. ${ }^{1,2}$ The annual costs of care for persons with AD severely tax the health care system today, and are predicted to singlehandedly create a future health care crisis.

$\mathrm{AD}$ is characterized clinically by the development of early amnestic and executive dysfunction, that eventually spreads across cognitive domains, that leads to the complete incapacity and development of end-stage dementia. ${ }^{3}$ The major pathological hallmarks of $\mathrm{AD}$ are extracellular amyloid $(\mathrm{A} \beta)$ plaque deposition and intraneuronal neurofibrillary tangle formation. ${ }^{4-6}$ Emerging evidence suggests that progressive inflammation and increased oxidative stress play a key role in the early development of such pathological features. ${ }^{7-12}$ Such mechanisms have also been clearly posited to play a key role in synaptic dysfunction and the loss of neuronal integrity that may precede the overt appearance of amyloid plaques and neurofibrillary tangles in the brains of affected individuals.

Cellular pathways involved in the homeostasis and regulation of brain fatty acids are central players in both the inflammatory and oxidative stress cascades implicated
Correspondence: Gregory A Jicha Sanders-Brown Center on Aging, $\mathrm{Rm}$ 223, 800 South Limestone Street, University of Kentucky College of Medicine, Lexington,

KY 40536-0230, USA

Fax + I 8592573819

Email gajich2@email.uky.edu 
in the pathogenesis of $\mathrm{AD}{ }^{7,8}$ The present review highlights the latest discoveries in the basic biology of omega-3 fatty acids in the brain in the animal model, in vitro, and human data, on the relationship of omega- 3 fatty acids with $\mathrm{AD}$, and clinical trial results using omega- 3 fatty acids for the treatment of AD. Furthermore, these findings are synthesized into a rationale strategy that may help guide the future use of omega-3 fatty acid supplementation in the management or prevention of early $\mathrm{AD}$.

\section{Fatty acids in the brain}

Fatty acids serve as both energy substrates and integral membrane components essential for proper neuronal and brain function. ${ }^{7-10,12-14}$ Triglycerides are the substrate for energy metabolism in low glucose states, while polyunsaturated fatty acids (PUFA) are integral membrane lipids that serve to maintain both the structure and function of neuronal membranes, membrane associated proteins and protein complexes. The incorporation of PUFA into neuronal membranes lowers the total cholesterol fraction, leading to increased membrane fluidity that is essential to maintain synaptic structures (Figure 1). ${ }^{15}$ As a result of PUFA incorporation increased membrane fluidity can increase the number and affinity of receptors in the synapse and improve neurotransmission. Such fluidity is important in promoting synaptic plasticity that is essential for learning, memory, and other complex cognitive processes.

PUFAs are also converted into essential membrane phospholipids and second messengers that modulate inflammation, oxidative stress, and neuronal health. ${ }^{7,8,10,12,13,16,17}$ Long chain PUFAs in the brain include both omega-3 PUFAs, (eg, docosahexanoic acid [DHA, 22:6n3]), and omega-6 PUFAs (eg, arachidonic acid [AA, 20:4n6]). PUFAs such as DHA and AA are created through hydrolysis mediated crosslinking (double bond formation) along the carbonyl backbone of their saturated fatty acid precursors. This chemical structure promotes increased stability and membrane fluidity that allows unique protein lipid interactions not otherwise afforded by saturated fatty acids or omega- 6 fatty acids such as AA and cholesterol. As such, PUFAs have been identified as integral
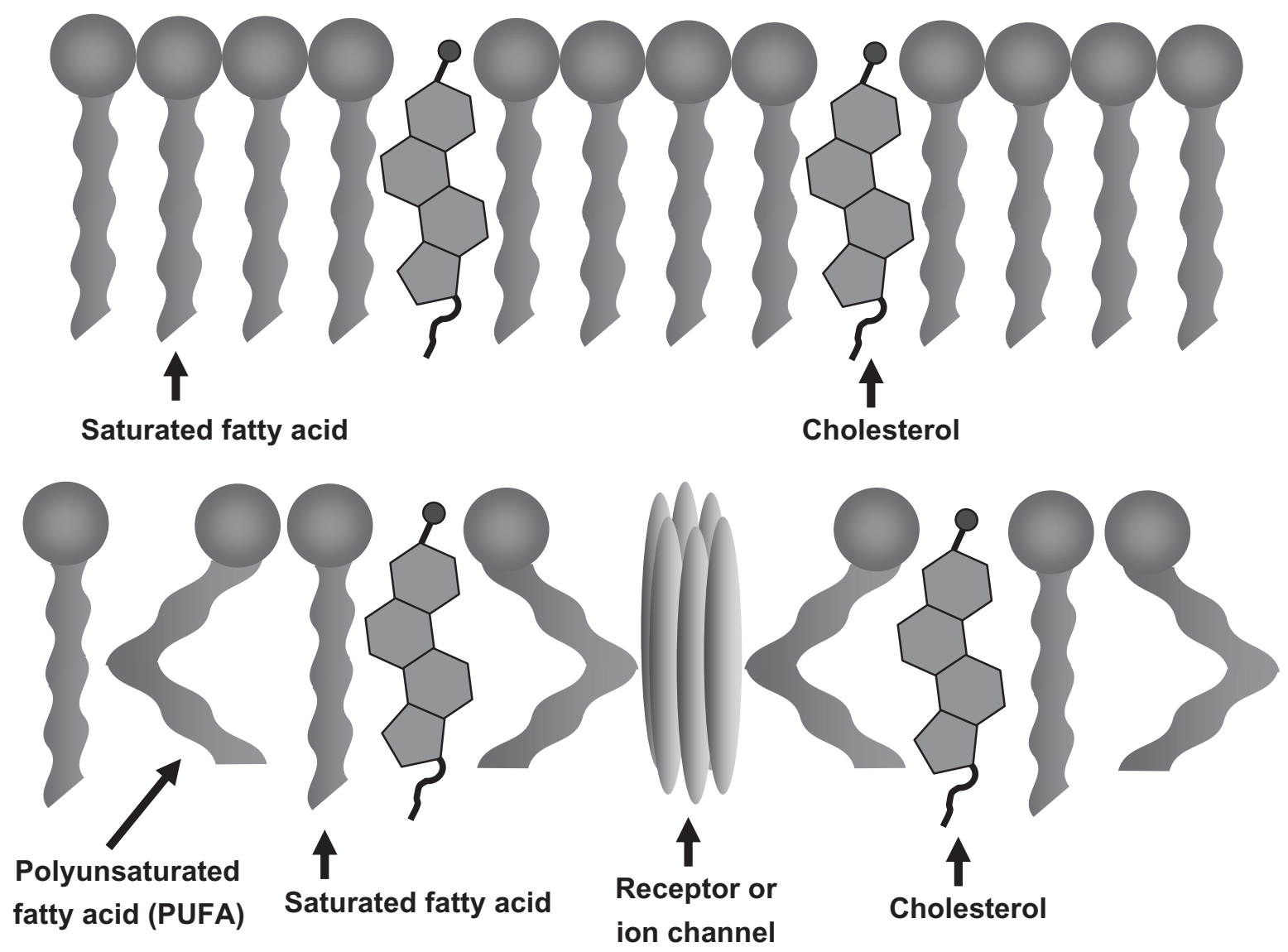

Figure I Polyunsaturated fatty acids increase membrane fluidity and facilitate incorporation of essential membrane bound proteins required for synapse and lipid raft formation A) Saturated fatty acids and cholesterol pack tightly to form a more rigid and less fluid lipid bilayer. B) Double-bond formation in polyunsaturated fatty acids creates a "bend" in the fatty acid tail that prevents the tight packing of saturated fatty acids and cholesterol leading to increased membrane fluidity and incorporation of integral synaptic proteins. 
membrane components required for lipid raft formation that is essential for membrane and membrane-bound protein transport, synapse formation, and neuronal membrane integrity. ${ }^{7-9}$ In contrast to AA, which is distributed equally across gray and white matter areas in the brain, DHA is found predominantly in neuronal membranes in the gray matter and constitutes the major omega-3 fatty acid in the brain. ${ }^{17}$

DHA may also play a key role in both the structure and function of brain regions involved in the formation of new memories. Hippocampal DHA levels have been directly associated with dietary intake, and higher levels have been shown to enhance hippocampal-dependent learning processes. ${ }^{18}$ DHA has also been linked to the recently described phenomena of hippocampal neurogenesis in the adult brain. ${ }^{19,20}$ The role of DHA in this process is modulated through the expression of basic helix-loop-helix transcription factors in neuronal stem cells originating in the dentate gyrus. ${ }^{21}$ Alterations in gene expression induced by DHA help to promote cell cycle exit and allow neuronal differentiation to occur. Enhanced neurogenesis in such regions has been postulated to lead to increased neuronal density and function in this critical circuitry for new memory formation. High dietary intake of omega-3 PUFAs has been directly associated with increased gray matter volume in corticolimbic circuitry that represents the affective input for memory formation and cortical arousal in the brain. ${ }^{22}$ Anterior cingulate, right amygdala and right hippocampal volumes were all correlated with increased omega-3 PUFA intake after correction for total brain volumes. ${ }^{22}$ The finding of selectivity of this effect for the right amygdala and hippocampus is unusual given that the proposed mechanisms for DHA augmentation of neurogenesis, new synapse formation, and increased gray matter volume should not be hemisphere dependent. ${ }^{19,21}$ This finding is intriguing given the recent neuroimaging data that suggest the asymmetric involvement of neuronal loss and degeneration in the hippocampus of research subjects with AD. ${ }^{23-27}$ Further work is clearly needed in this area before definite conclusions can be drawn.

DHA is considered an essential PUFA as de novo production is not possible in humans. Eicosapentanoic acid (EPA, 20:5n3) and $\alpha$-linoleic acid (ALA, 18:3n3) can be converted to DHA by some cell types and organ systems in the body, however, there is great variability in the degree to which this can be accomplished in humans. While ALA can be derived from terrestrial plants and is commonly found in a Western or terrestrial-based diet, DHA and EPA, initially created by photosynthetic microalgae, are exclusively derived from marine animals. Little EPA or ALA is found in the brains of humans, again attesting to the importance of DHA in the maintenance of neuronal membrane integrity and the signaling cascades related to PUFAs in the brain. Ratios of omega-6 to omega-3 PUFAs may be as important as absolute levels of these lipids in the homeostasis of central nervous system (CNS) inflammation and oxidative stress. Induction of the pro-inflammatory AA cascade of signaling events exerts a negative influence on the downstream oxidative stress modulation cascades dependent on DHA and its derivatives, and vice versa. It is intriguing that the dramatic increase in the prevalence of $\mathrm{AD}$ over the last century not only parallels the increase in average lifespan, but also an increase from 2 to more than 20 of the ratio of omega- 6 to omega-3 PUFAs in the average Western diet. ${ }^{17,28}$

While much is known about the role of AA in the generation of the eicosanoids including the prostaglandins by cyclooxygenases $(\mathrm{COX})$ and their role in initiating and maintaining the inflammatory cascade in the brain and other tissues, the critical role(s) of DHA and other omega-3 PUFAs are just beginning to be understood. ${ }^{10,12-14,17,28}$ Analogous to the formation of the eicosanoids from COX actions on AA, DHA is modified through phospholipase $\mathrm{A}_{2}$ and lipoxygenase to form the docosanoid, neuroprotectin 1 (NPD1, see Figure 2). ${ }^{13,29,30}$ Several studies have demonstrated reduced activity of phospholipase $A_{2}$ in brain tissue, cerebrospinal fluid CSF and platelet fractions from AD subjects. ${ }^{31-36}$ Such alterations in this critical enzyme required for the production of NPD1 may play a significant role in the development of AD. The formation of NPD1 from DHA is tightly regulated by the redox state of neurons. Increased oxidative stress caused by $\mathrm{A} \beta$ exposure, hypoxia, and interleukin (IL)- $1 \beta$ activity upregulates phospholipase $A_{2}$ generating NPD1, which exerts a powerful negative feedback mechanism to control excessive oxidative stress. The actions of DHA and NPD1 additionally include upregulation of the Bcl-2 family of anti-apoptotic proteins, down regulation of pro-apoptotic signaling pathways, and inhibition of the pro-inflammatory, COX-mediated, production of eicosanoids, including prostaglandin synthesis from AA, which can further contribute to neuronal injury and destruction in degenerative diseases such as AD. Further elucidation of the molecular mechanisms through which increases in NPD1 and other metabolites of DHA enhance neuronal health is important for understanding how decreased levels of these important signaling molecules may contribute to the pathogenesis of AD and other degenerative diseases.

PUFAs are powerful antioxidants that are also readily oxidized in a nonenzymatic fashion when subjected to excessive 


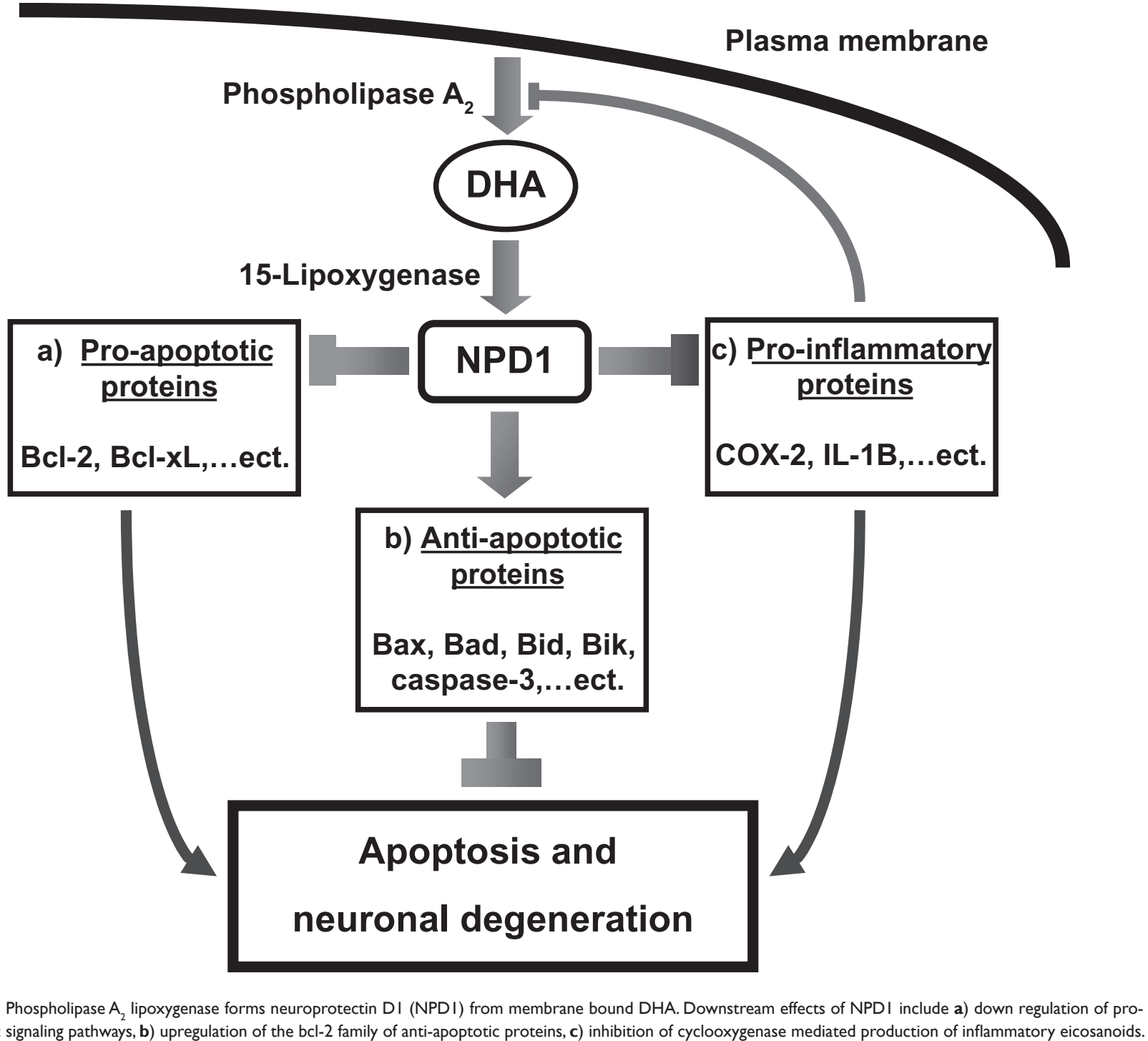

apoptotic signaling pathways, b) upregulation of the bcl-2 family of anti-apoptotic proteins, c) inhibition of cyclooxygenase mediated production of inflammatory eicosanoids.

neuronal oxidative stress. ${ }^{12}$ Nonenzymatic oxidation of AA results in the formation of the biologically active hydroxynonenal $\mathrm{F}_{2}$ isoprostanes ( $\mathrm{F}_{2}$ IsoPs). ${ }^{12,37} \mathrm{DHA}$ is similarly oxidized to the biologically active hydroxyhexenal $\mathrm{F}_{4}$ neuroprostanes $\left(\mathrm{F}_{4} \mathrm{NPs}\right)$, so named because of their exclusive reflection of the redox state of neuronal populations in the brain. ${ }^{12,13}$ Early studies showed that $\mathrm{F}_{2}$ IsoPs and $\mathrm{F}_{4} \mathrm{NPs}$ were increased in the brains and ventricular fluid of autopsied, late stage, $\mathrm{AD}$ patients compared with controls. ${ }^{38-43} \mathrm{~A}$ later study showed increased $\mathrm{F}_{2}$ IsoPs and $\mathrm{F}_{4} \mathrm{NPs}$ in the brains of mild cognitive impairment (MCI) patients compared to normal controls. ${ }^{11}$ This study indicates the oxidative damage of AA and DHA is an early event in the pathogenesis of AD. The $\mathrm{F}_{2}$ IsoPs, $\mathrm{F}_{4} \mathrm{NPs}$, and other oxidative derivatives of PUFAs not only serve as important markers of oxidative stress in neurodegenerative diseases such as $\mathrm{AD}$, they also adopt new biological activities and certain derivatives can enhance $A \beta$ oligomerization in vitro. The usefulness of assays for $\mathrm{F}_{2}$ IsoPs and $\mathrm{F}_{4} \mathrm{NPs}$ as biomarkers for therapeutic trials investigating PUFA and other antioxidative compounds may prove valuable to assess the biological efficacy of such strategies irrespective of the outcome of primary clinical endpoints.

\section{DHA and $A \beta$}

The amyloid cascade hypothesis of AD pathogenesis holds as a basic tenet the early formation of toxic amyloid-beta (A $\beta$ ) fragments from the normal cellular, transmembrane, amyloid precursor protein (APP). ${ }^{44,45}$ Release of toxic A $\beta$ fragments leads to the early formation of soluble dimeric and oligomeric aggregates, that directly inhibit synaptic function and are highly toxic to neurons. ${ }^{46}$ Further aggregation leads to the formation of insoluble macromolecular complexes that 
deposit in the extracellular milieu of the brain parenchyma to form amyloid plaques, a key pathological hallmark of AD. The combination of the toxic effects of both early soluble oligomers and insoluble plaque deposits of $A \beta$ leads to further neuronal disruption and eventual cell death which in turn is responsible for the irreversible cognitive decline found in AD.

APP is normally processed by two membrane-associated, catalytic proteases, $\alpha$ - and $\beta$-secretase. Such proteolytic cleavage leads to the formation of harmless peptide fragments that are readily degraded further or shed from the CNS. In AD, a third membrane associated protease, $\gamma$-secretase, cleaves APP within the transmembrane portion of the protein. This cleavage combined with the extracellular cleavage of APP by $\beta$-secretase, which appears to be the rate-limiting enzyme in this degenerative pathway, releases the toxic $\mathrm{A} \beta$ peptide in a characteristic 40 or 42 amino acid form. ${ }^{47-51}$ While amyloid plaques are composed predominantly of 42 amino acid fragments, the 40 amino acid fragment appears to accumulate in small arterioles and capillaries in the brain leading to cerebral amyloid angiopathy, increased risk of cerebral hemorrhage and the impairment of nutrient exchange, that all have a potential role in reducing the transport of essential PUFAs across the blood brain barrier. Such a role is speculative at present and deserves further study.

A wealth of data collected from in vitro assays, cell culture systems, and transgenic animal models of AD supports a direct association of omega-3 PUFAs, especially DHA, with amyloid processing in the brain. ${ }^{9,10,52-55}$ Omega-3 PUFAs as integral membrane components may act to alter amyloidogenic processing in several distinct and possibly interrelated ways including: a) facilitate the interaction of $\alpha$-secretase with APP to produce nontoxic fragments and prevent the formation of $A \beta ; b$ ) shield the essential recognition sequence and intramembrane cleavage site for $\gamma$-secretase; c) serve as a local sink for free radicals that reduce the enzymatic augmentation of $\gamma$-secretase activity, that can be induced by free radical damage to the protein complex, which is important for the regulation of normal $\gamma$-secretase function; d) directly inhibit fibrillation and formation of toxic oligomeric species of $A \beta$ (Figure 3). . $^{7,10,52,53}$

Cell culture studies consistently demonstrate a lowering of $\mathrm{A} \beta$ production by $20 \%$ after treatment with DHA. ${ }^{13,56}$ The activities of both $\beta$ - and $\gamma$-secretase are down-regulated in DHA treated cultures and these effects appear to be directly related to the composition of lipid rafts that allow both the association and regulation of these critical enzymes in the formation of toxic $A \beta{ }^{9}{ }^{9,13,54,57}$ Additional studies have demonstrated increases in anti-apoptotic proteins of the Bcl-2 family, translocation and phosphorylation of Akt, and down-regulation of caspase- 3 and
9 in DHA cultured neurons, which are likely mediated by the formation of NPD1 (as described above). ${ }^{9,13}$ Such downstream effects of DHA supplementation are highly desirable in the interference of cellular mechanisms suggested to play a role in the pathogenesis of AD.

Transgenic animal models that develop amyloid plaque and vascular $A \beta$ deposits allow further study of the potentially beneficial effects of omega-3 PUFA supplementation in the abrogation of AD-like pathology. Studies have been done in several different model systems including the widely used and well characterized Tg2576, ${ }^{58,59}$ APPswe/PS1dE9, ${ }^{56}$ and 3xTg-AD mice ${ }^{55}$ (Frank LaFerla's triple transgenic mice). Nontransgenic mice infused with $A \beta$ have also been used as a model system to investigate the beneficial effects of omega-3 PUFA supplementation. ${ }^{60-62}$ The experimental paradigm for treatment is as easy as altering the fatty acid composition of the diet used to feed the animals. A universal finding in every study using amyloid producing transgenic mice to date is a significant decrease in A $\beta 42$ levels, with several studies also demonstrating decreases in $A \beta 40$ and total A $\beta$ levels. Tg2576 mice treated with omega-3 PUFA supplementation have universally shown reduced plaque numbers, while APPswe/PS1dE9 mice have not, despite the finding of reduced $A \beta$ in these animals. A complete explanation for this discrepancy has not been reported, but may be related to the more aggressive time course of A $\beta$ deposition in the APPswe/PS1dE9 mice (6 months) compared to the later development of amyloid pathology in Tg2576 mice (9-12 months). Green and colleagues demonstrated decreased presenilin 1 ( $\gamma$-secretase) in the triple transgenic mice as a result of DHA supplementation. ${ }^{55}$ Calon and colleagues investigated apoptotic regulators and found decreases in bipolar affective disorder and increases in PI3 and Akt kinase pathways. ${ }^{58}$ Alterations in lipid raft and membrane composition as well as fluidity of the membrane in synaptosomal preparations have been well characterized in the $A \beta 40$-infused Wistar rats. ${ }^{60,63}$

Comparing the studies is difficult due to the use of different dosing regimens, variations in treatment length, heterogeneity in animal model system, and outcome measures. Despite these problems and caveats, these animal models have proved useful and provide valuable insights into the effects of omega-3 PUFA supplementation on the development of $\mathrm{AD}$-like pathologic features, formation of $\mathrm{A} \beta$ fragments, oxidative stress markers, secretase activity, regulation of pro- and anti-apoptotic proteins, membrane fluidity, and lipid raft composition, and performance on behavioral tasks such as the Morris water maze. 


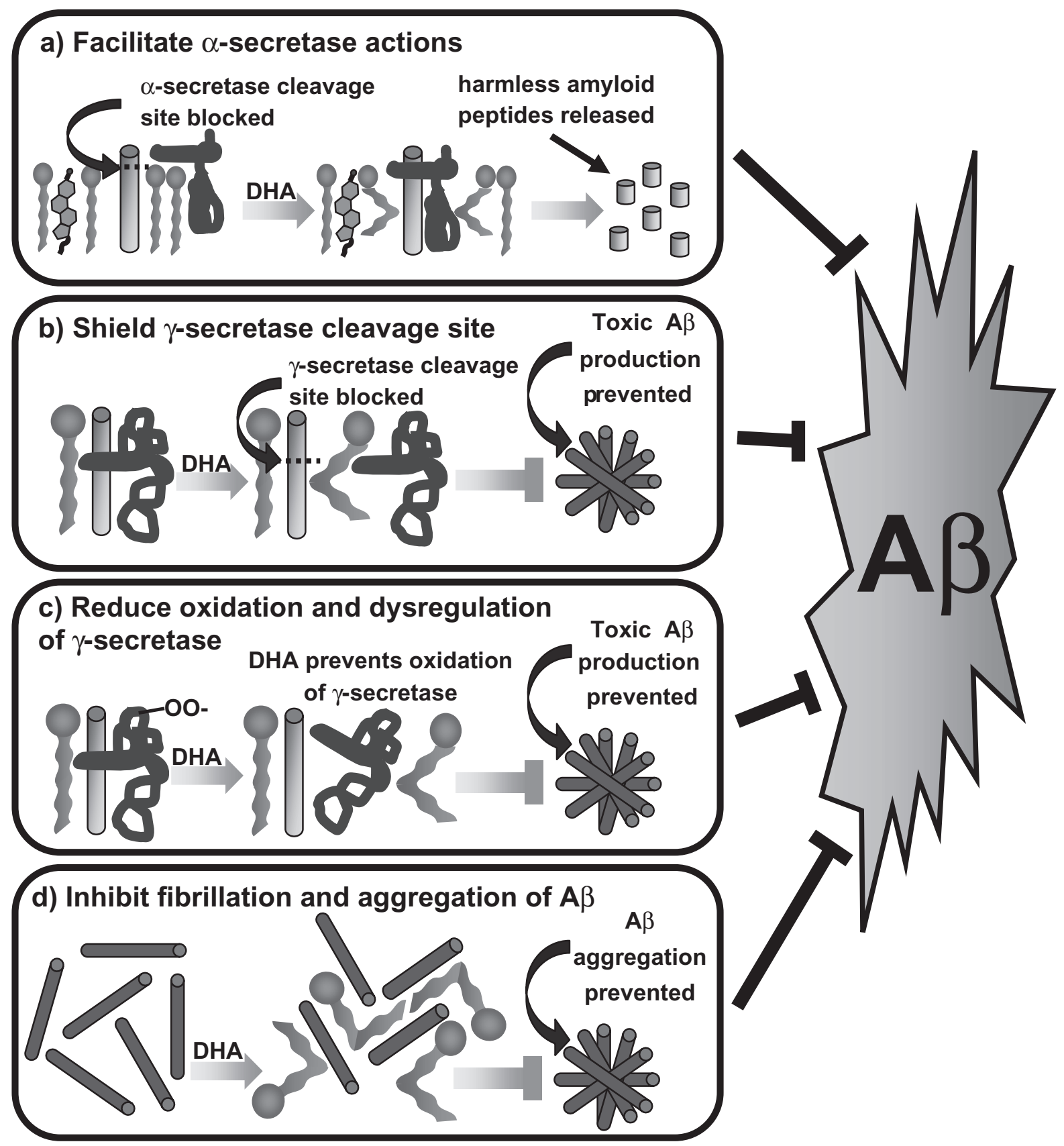

Figure 3 Omega-3 PUFAs influence amyloidogenic processing through several distinct and interrelated mechanisms: a) facilitation of the interaction of $\alpha$-secretase with APP to produce non-toxic fragments and prevent the formation of $A \beta, \mathbf{b}$ ) shielding the essential recognition sequence and intramembrane cleavage site for $\gamma$-secretase, $\mathbf{c}$ ) serving as a local sink for free radicals that reduce the enzymatic augmentation of $\gamma$-secretase activity that can be induced by free radical damage to the protein complex important for the regulation of normal $\gamma$-secretase function, and d) directly inhibiting fibrillation and formation of toxic oligomeric species of $A \beta$.

\section{PUFA, cholesterol, and}

\section{cerebrovascular disease contributions to AD}

The links between cerebrovascular disease (CVD) and AD drawn from studies of comparative risk factors are extensive and have led to several interventional studies that hypothesize that AD may be a "vascognopathy". ${ }^{64-68}$
The interplay between PUFAs, cholesterol, CVD, and their interrelationship with the development of $\mathrm{AD}$ is a topic of extreme interest in the field. While many important factors are associated with both CVD and $\mathrm{AD}$, this review will focus on the interrelationships of omega-3 PUFAs, cholesterol, and apolipoprotein E (ApoE) status exclusively. Several excellent reviews and abundant primary literature exist on the interplay and synergism between CVD and AD 
mechanisms of neurodegeneration. The beneficial effects of omega-3 PUFAs in preventing CVD have been largely attributed to its role in interference with platelet aggregation, lowering cholesterol, and preventing atherosclerotic buildup in vulnerable blood vessels.

Omega-3 PUFAs, especially EPA, although not DHA, inhibit platelet aggregation and serve as effective anticoagulants. ${ }^{69}$ These properties give rise to the major risks of omega-3 PUFA supplementation that include an increased risk of bleeding diathesis, especially for those taking medications that further interfere with platelet aggregation or the clotting cascade. ${ }^{16,70-76}$ While this property of EPA may be seen as deleterious, it is possible that antiplatelet activity could be responsible for the beneficial effects of omega-3 PUFA supplementation reported in many studies. There is often a discrepancy in the effect of omega-3 PUFA supplementation in humans based on the source such as pure DHA or fish oil products which include a combination of both DHA and EPA. Such disparate data could suggest that the antithrombogenic properties of EPA allow increased blood flow and supply of nutrients as well as increased removal of toxic metabolites and proteins from the brain that might otherwise augment the degeneration characteristic of AD. Definitive studies investigating such mechanisms are lacking currently but deserve further exploration.

The deleterious effects of cholesterol on cardiovascular health can be largely attributed to stimulation of atherogenesis, yet cholesterol serves several important functions and is an essential component of cellular membranes. The brain is the most cholesterol-rich organ in the human body. Cholesterol is not transported into the brain, as are fatty acids, but instead it is synthesized in the brain, largely within astrocytes. Like omega-3 PUFA, cholesterol can be oxidized, but this is dependent on enzymatic rather than non-enzymatic pathways including the CYP46 system. Oxidized cholesterol is relatively stable compared to the high chemical reactivity of $\mathrm{F}_{2}$-IsoPs and $\mathrm{F}_{4}$-NPs. Cholesterol is also readily eliminated through the blood brain barrier in an oxidized form, preventing its buildup and further oxidative damage. Cholesterol may directly increase the activity of $\beta$ - and $\gamma$-secretases. ${ }^{77,78}$ High cholesterol exposure increases $A \beta$ production, whereas low cholesterol exposure decreases $A \beta$ production in cell culture and in transgenic mouse models of AD. The interplay between cholesterol and omega-3 PUFA in membrane stabilization and fluidity as well as in the development of atherosclerosis appears to be a critical factor influencing the development of AD.

Apolipoprotein E (ApoE) is the major carrier of cholesterol in the brain. The $\varepsilon 4$ allele of ApoE is the most potent risk factor for sporadic, late-onset $\mathrm{AD}$ characterized to date ${ }^{67,79-82} \mathrm{ApoE}$ is produced in astrocytes and its production is upregulated during development and regeneration of the CNS. ApoE is also directly implicated in the mediation of $\mathrm{A} \beta$ production. There are three primary alleles for the $\mathrm{ApoE}$ gene, $\varepsilon 3$ which is the most common and neither imparts risk nor protection, $\varepsilon 2$ which appears protective against $A D$, and $\varepsilon 4$ that is associated with earlier onset and development of AD. ApoE e 4 is a risk factor for increased serum cholesterol in both humans and animals. This effect can augment the production of toxic $A \beta$ as described above. In addition, cholesterol promotes atherogenesis, which is directly related to the development of $\mathrm{AD}$ pathology when present in the circle of Willis. ${ }^{83-86} \mathrm{ApoE}$ has also been shown to enhance in vitro oligomer and fibril formation from $\mathrm{A} \beta$ monomers. ${ }^{87,88}$ Direct interactions between ApoE and omega-3 PUFAs have not been fully elucidated. The interplay between omega-3 PUFAs and ApoE may be mediated by cholesterol levels and regulatory mechanisms rather than through a direct interaction.

Clearly, the relationship between CVD and AD, including the association of both high cholesterol levels and the presence of the ApoE $\varepsilon 4$ allele, and omega-3 PUFAs deserves further study and should not be dismissed in favor of the alternative hypothesis that omega-3 PUFAs directly influence $A D$ through reduction of oxidative stress or alterations in $\mathrm{A} \beta$ metabolism as described above.

\section{Cross-sectional, epidemiological, and prospective cohort studies}

Several studies of dietary intake of PUFAs have been published in large cohorts of individuals with AD worldwide and demonstrate a largely positive effect of increased dietary intake with a reduced risk of $\mathrm{AD}$ (Table 1). This has been reviewed in part by Cole and colleagues and again by Cunnane and colleagues ${ }^{7,8}$ The weaknesses of these studies lie largely in the retrospective nature of the data collection and the confound of reporter bias in estimating dietary intake of PUFAs from food-frequency questionnaires (FFQ). Still several of these studies have validated their findings with quantitative measures of fatty acid composition in serum. Others have looked at prospective conversion to dementia that further reinforces the potential beneficial effects of PUFAs in slowing or preventing $\mathrm{AD}$ conversion. A summary of the relevant findings from these studies and their inclusion of data on confounding variables are presented below.

In 1997, Kalmijn and colleagues published data from the Rotterdam Study that included 5,386 individuals that were non-demented at baseline. ${ }^{89}$ All completed a FFQ as 


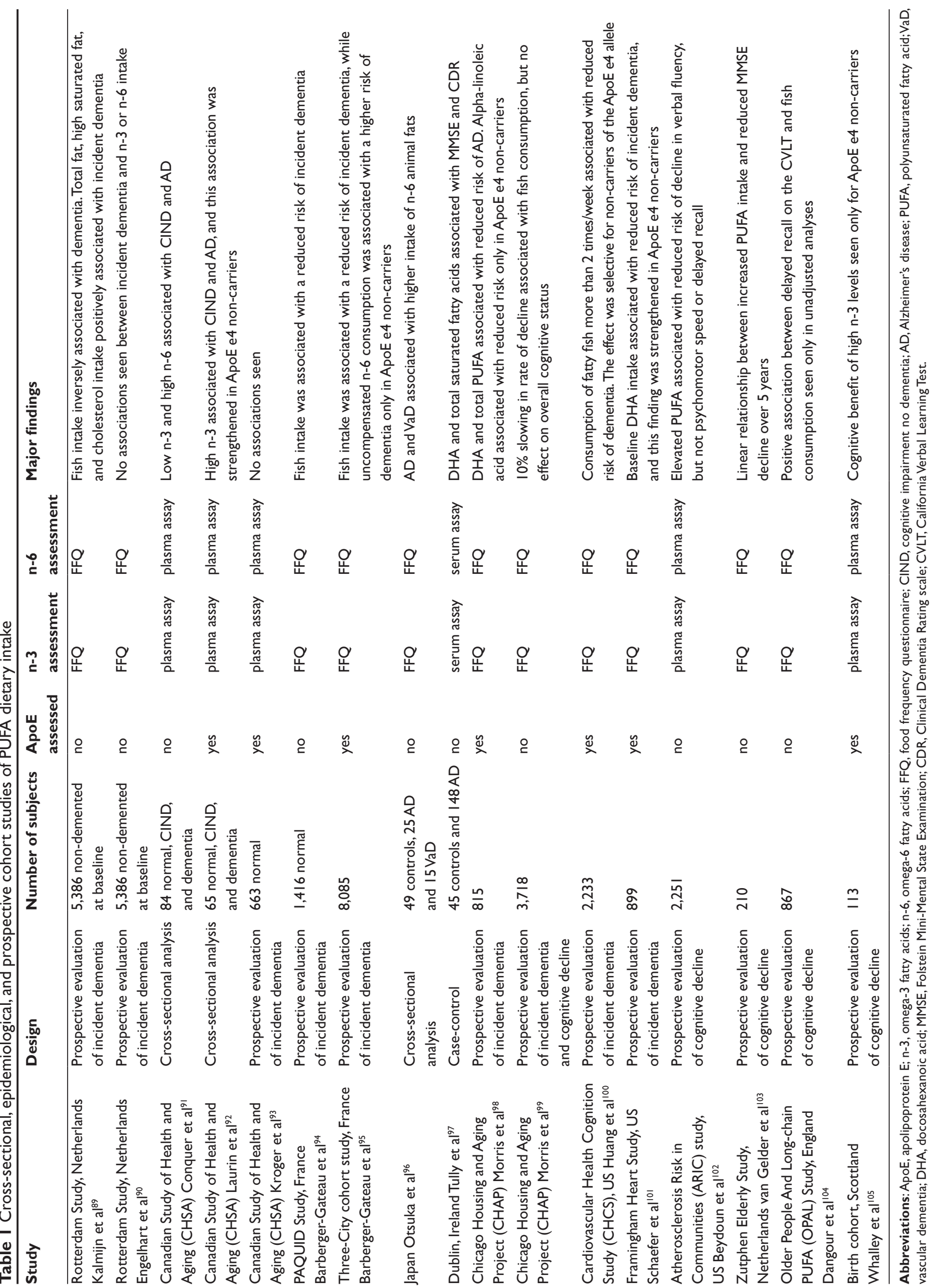


part of their baseline evaluation. High total fat, saturated fat, and cholesterol were associated with an increased risk of dementia, whereas fish consumption was inversely related to both all-cause dementia and more strongly with incident AD. High total and saturated fat intake was especially associated with vascular contributions to dementia in this study and was rigorously assessed with MRI. Specific quantitative measures of cholesterol and serum fatty acid profiles or ApoE genotype were not routinely performed or reported on in this study. The duration of the follow-up was quite short at 2.1 years.

The Rotterdam study re-examined their data in 2002 to include a longer follow-up period and increased the numbers of subjects with incident AD in this period ( $n=197$ of 5,396 normal subjects at baseline). ${ }^{90}$ This study failed to replicate their original findings. No association between dietary intake of total, saturated, or trans-fat, cholesterol, omega-3 or omega-6 PUFA was found in this analysis.

The Canadian Study of Health and Aging (CHSA) rigorously examined plasma fatty acid profiles cross sectionally in a small cohort of 84 subjects in 2000. ${ }^{91}$ Plasma total PUFA, DHA, and n-3/n-6 ratios were lower in $\mathrm{AD}$, other dementia, and individuals with cognitive impairment that were not demented (CIND). Omega-6 fatty acids were higher in AD and CIND cases than in normals and non-AD dementias. The authors concluded that low plasma PUFA levels may be a risk factor for all cause dementia. This study provided the first look at n-3/n-6 ratios and demonstrated a rigorous quantitative application of methodology to study the association of cross sectional PUFA levels with cognitive status, albeit in a small select sample of the larger CHSA population.

The data were reanalyzed in 2003 including only a subset of the original 84 subjects in their earlier report $(n=65) .{ }^{92}$ Included for the first time was an analysis of the potential effects of the ApoE $\varepsilon 4$ allele in modulating the association of plasma PUFA concentrations and clinical status. While the study reported a lack of association of PUFA with cognitive status in the cross sectional analysis, EPA levels were reportedly higher than controls in CIND, and DHA and total PUFA were higher in dementia cases in the prospective analysis. This association was strengthened in the subset on subjects that were not carriers of the ApoE $\varepsilon 4$ allele. No association of ApoE status with PUFA concentrations was seen in normal control or CIND subjects.

In 2009, the CHSA published an updated report in 663 cognitively normal subjects at baseline, followed prospectively that included 149 incident cases of dementia at study end. ${ }^{93}$ This study again failed to show a significant association of baseline plasma PUFA levels and risk of incident cognitive decline in this population. These effects were not altered by covariate adjustment for potential confounders such as ApoE status.

Barberger-Gateau and colleagues reported their findings from the PAQUID study in France in 2002. ${ }^{94}$ An FFQ was administered to 1674 subjects during the third wave of the study. Participants were followed up to 2, 5, and 7 years and 1,416 had at least one follow-up visit revealing 170 incident cases of dementia. Fish and seafood consumption at baseline was inversely associated with risk of incident dementia. Other meat intake showed no benefit or a deleterious effect. Education confounded these results suggesting that the effect might be simply explained by higher educational status or that higher educational status was simply associated with higher fish and seafood consumption. This is an important consideration as education is a well recognized mediator of cognitive decline. The methods for this study lacked rigor and the findings were speculative at best.

Barberger-Gateau and colleagues evaluated dietary patterns much further by using data from the larger Three-City cohort Study in $2007 .{ }^{95}$ A total of 8,085 subjects from Bordeaux, Dijon, and Montpellier, France, who had at least one follow-up visit were included in the analysis. The authors found that weekly fish consumption was associated with a reduced risk of AD and all cause dementia only in ApoE e4 non-carriers. Regular consumption of omega- 6 rich foods that was not balanced or compensated by omega- 3 rich foods was associated with an increased risk of all cause dementia only among ApoE e4 non-carriers. This study importantly, re-emphasized the links between the potential benefits of omega-3 rich diets, dementia, and the relationship between omega- 3 and omega6 fatty acid intake.

A small study from Japan was published in 2002 that included 25 patients with $\mathrm{AD}, 15$ patients with vascular dementia (VaD) according to NINCDS-AIREN criteria, and 49 controls. ${ }^{96}$ Data were assessed from the administration of a standardized FFQ. This study demonstrated that energy intake exceeded demands in the dementia groups compared to controls. The dietary intake of $\mathrm{AD}$ and $\mathrm{VaD}$ subjects was similar with the exception of higher intake of animal fats in the $\mathrm{AD}$ vs $\mathrm{VaD}$ groups. This study reinforced the importance of low n-3/n-6 ratios in the development of dementia in Japan.

In 2003, researchers from Dublin Ireland presented a case-control study on 148 AD subjects and 45 cognitively normal controls. ${ }^{97}$ Applying the rigorous methodology of the CHSA study of 2000, the researchers relied on serum measurements of EPA, DHA, and total serum saturated 
fatty acids. While all three measurements were significantly reduced in $\mathrm{AD}$ versus normal control sera in adjusted analyses, only DHA and total serum saturated fatty acids were associated with Mini-Mental State Examination (MMSE) and Clinical Dementia Rating scale (CDR) scores in step-wise multiple regression analyses.

Morris and colleagues presented data from a FFQ administration to 815 subjects in the Chicago Housing and Aging Project (CHAP) in 2003. ${ }^{98}$ The study concluded that participants who consumed fish once a week or more had a $60 \%$ reduced risk of $\mathrm{AD}$ compared to those who rarely, or never, ate fish. Total PUFA and DHA, but not EPA intake was associated with this reduced risk for AD. Intake of ALA, a precursor to DHA derived from plant rather than fish sources, was also protective, but only in ApoE $\varepsilon 4$ carriers, in contrast to the positive effects of PUFA seen only in ApoE $\varepsilon 4$ carriers in the CHSA study above. The reasons for this discrepancy were not explored further.

The CHAP study re-examined their data in 2005 with a larger cohort of 3,718 subjects that had completed at least two cognitive assessments. ${ }^{99}$ This analysis failed to confirm the previously robust findings of the initial analysis, but did again show a small effect of fish consumption which favored a $10 \%$ slowing of the rate of cognitive decline in subjects but did not appear to affect the cognitive state for individuals studied. ApoE status was not studied as a covariate in this iteration of the CHAP study data.

The Cardiovascular Health Cognition Study directly examined the association of fish intake (lean versus fatty) with dementia, $\mathrm{AD}$, and $\mathrm{VaD}$ in relation to $\mathrm{ApoE}$ status in 2,233 subjects in their 2005 report. ${ }^{100}$ The authors reported that while consumption of lean fried fish had no protective effect, the consumption of fatty fish more than twice a week was associated with a reduced risk of dementia and AD. Stratification by ApoE $\varepsilon 4$ demonstrated that this effect was selective for non-carriers of the $\varepsilon 4$ allele only.

The Framingham Heart Study examined plasma DHA levels at baseline in 899 subjects followed prospectively for a mean of 9.1 years. ${ }^{101}$ Over the study period, 99 incident cases of dementia were observed and increased baseline DHA levels were associated with a reduced risk of incident dementia in this cohort. Adjustment for ApoE status increased the level of significance for this finding in this cohort composed of approximately $78 \%$ ApoE $\varepsilon 4$ non-carriers.

The Atherosclerosis Risk in Communities (ARIC) study prospectively studied the association between plasma fatty acids and cognitive decline in 2,251 subjects enrolled between 1990-1992 and 1996-1998. ${ }^{102}$ Elevated PUFA levels were associated with reduced risk in decline in verbal fluency. No associations between PUFA levels and psychomotor speed or delayed recall were found. The association of reduced risk in verbal fluency decline with elevated PUFA levels was augmented in subjects who were hypertensive or hyperlipidemic. ApoE status was not a significant covariate in this analysis.

The Zutphen Elderly Study used a FFQ to assess dietary status of 210 participants from the Netherlands in 2007 and related this to subsequent 5-year cognitive decline as measured by the MMSE. ${ }^{103}$ This study demonstrated significantly less cognitive decline in fish consumers than non-consumers over the 5-year follow-up period. A linear relationship was found for intake of total PUFA and cognitive decline, with an average difference of 1.1 points decline on the MMSE per increased $380 \mathrm{mg} /$ day of total PUFA in the diet.

The Older People and n-3 Long chain polyunsaturated fatty acid (OPAL) study examined 867 persons aged between 70-79, without dementia, an MMSE score of 24-30, and not taking regular fish oil supplements. ${ }^{104}$ The study design was a cross sectional analysis of self-reported fish consumption and an extensive battery of neuropsychological test measures. While unadjusted analysis of these data revealed significant positive correlations between fish intake and delayed recall on the California Verbal Learning Test, after adjustment for age, gender, education and general health status, no significant association was seen. ApoE genotype was not reported, nor was an assessment of n- 6 intake performed or considered as part of the analysis.

In 2008, Whalley and colleagues published their analysis of n-3 fatty acid erythrocyte membrane content and cognitive variation in 113 cognitively normal volunteers enrolled in a birth cohort study in Scotland and followed over three longitudinal assessments at ages $\sim 64,66$, and 68 years. ${ }^{105}$ The study was designed to test the observation of Huang and colleagues, ${ }^{100}$ that ApoE status was an important determinant in modulating the effect of n-3 intake on cognitive status. Fish oil supplement use was equally distributed between ApoE e4 carriers and non-carriers. While cognitive benefits were associated with higher erythrocyte n-3 content, this association was only significant in ApoE e4 non-carriers. The authors proposed a gene $\times$ environment interaction for cognitive aging and Alzheimer's disease (AD) based on their findings.

The weaknesses of the above studies lie in the variable assessment of potentially significant confounders such as ApoE status and dietary intake of omega-6 PUFAs, saturated or trans- animal fats. Only 59\% (10 out of 17 total studies) of the studies reported above assessed such confounds. Despite 
this caveat, $70 \%$ (7 out of 10) of the studies that assessed ApoE status and $71 \%$ (5 out of 7 ) studies that assessed dietary n-6/saturated/trans fats found a significant impact of such confounders on the association of PUFA status with cognitive decline or dementia.

To place the above studies in the appropriate context of whether omega-3 PUFA supplementation should be pursued as a plausible therapy to treat or prevent $\mathrm{AD}$, several points need to be considered. Of the population-based or cohort studies, 14 out of 17 showed a significant benefit of elevated omega-3 PUFA intake or measured plasma/serum levels in reducing the association with prevalent dementia, risk of cognitive decline, or the development of incident dementia. Two of the three studies that failed to show such an effect are from the CHSA cohort. The third showed a positive effect suggesting that either: 1) sampling bias; 2) methodological variability; or 3) marginal effects that straddle the level of significance are seen in this select study. In addition, 13 of the 16 studies used prospective follow-up of baseline data to examine the risk of incident cognitive decline or dementia rather than simply assessing a baseline association. Such strategies serve to further strengthen the association of omega-3 PUFA intake or plasma levels with cognitive status. The overwhelming data presented above favor a positive effect of omega-3 PUFA levels on cognitive status.

Despite the strengths of much of this data, the timing and duration of n-3 supplementation have yet to be determined. Such population and cohort based studies cannot take into account the possible need for more prolonged or lifelong dietary habits to define a benefit or association of n-3 supplementation with cognitive outcomes in the elderly. Such epidemiologic evidence for the prevention of cognitive decline and the development of dementia has been demonstrated previously for both estrogen replacement therapy and non-steroidal anti-inflammatory use. Yet the prospective, randomized, clinical trials clearly demonstrated an increased risk rather than the postulated benefit related to duration of treatment. Evidence that dietary modification, or the use of n-3 supplements, to prevent cognitive decline or dementia is beneficial for the at-risk elderly population will require rigorous assessment in prospective, randomized, clinical trial designs.

\section{Clinical trials of PUFA supplementation}

Only 6 prospective studies of PUFA supplementation have been completed to date in the area of cognitive decline and dementia. Four of these are published and the most recent two have been presented in abstract form at the 2009 International Conference on Alzheimer's Disease.

The first published study in 2004 included only 20 AD patients treated with $500 \mathrm{mg}$ ethyl-EPA twice a day for 12 weeks. ${ }^{106}$ This small sample size, short trial showed no effect on cognitive decline as would be expected given the study design. A significant increase in plasma and erythrocyte membrane EPA and total n-3 levels was demonstrated. No follow-up studies have been reported, and the relevance of EPA supplementation to brain health remains unclear. This study clearly demonstrated however that dietary supplementation has a direct impact on circulating plasma levels of PUFAs.

A second study from Japan, published in 2006, examined the effects of $240 \mathrm{mg} /$ day AA and DHA and colleagues $240 \mathrm{mg} /$ day olive oil in 21 randomized subjects with MCI (12 assigned to active study treatment and 9 assigned to the olive oil placebo). ${ }^{107}$ Ten subjects with organic brain lesions (cerebral infarction, hemorrhage, or traumatic brain injury greater than five years earlier), and 8 subjects with AD served as additional control groups and were not randomized. The subjects were followed over a period of 90 days of treatment and assessed before and after intervention. Primary outcome measures included the subtests of the repeatable battery for the assessment of neuropsychological status (RBANS). The MCI subjects randomized to active treatment showed an improvement on immediate memory and attention subtests. Subjects with organic brain lesions also improved in immediate and delayed memory test scores. Placebo-treated MCI and actively treated AD subjects showed no significant improvements in any of the RBANS subtests. The authors concluded that AA and DHA supplementation may be beneficial in persons suffering from cognitive decline due to organic brain damage or age-related cognitive decline. This study showed promise but clearly suffered from major methodological flaws in study design, and so the conclusions drawn are subject to careful scrutiny and review.

In 2006, the first large-scale, well-designed, randomized, double-blind, placebo-controlled trial of omega-3 PUFA supplementation was reported by Freund-Levi and colleagues. ${ }^{108}$ This study included 204 subjects with mild to moderate AD receiving a cholinesterase inhibitor randomized to treatment with $1.7 \mathrm{~g}$ DHA and $0.6 \mathrm{~g}$ EPA per day or placebo for 6 months and included a 6-month open label extension in which all subjects received the omega-3 PUFA treatment. Primary outcome measures included the MMSE and Alzheimer's Disease Assessment Scale cognitive component (ADAS$\operatorname{cog}$ ). No significant differences in these primary outcome 
measures were observed between treated and placebo groups, however a subgroup analysis of mildly affected individuals (MMSE $>27, \mathrm{n}=32$ ) demonstrated a significant reduction in MMSE decline compared to the placebo subgroup. Similar effects were seen with active treatment in the 6-12 month period for the subjects with MMSE $>27$ treated initially with placebo. Of note, only $31 \%$ of the study cohort were ApoE $\varepsilon 4$ non-carriers and the treatment effect of ApoE status was not assessed as the groups were matched in this regard. The authors concluded that the beneficial effects of omega-3 PUFA supplementation may be dependent on cognitive status at time of treatment intervention with those in the earliest stages of disease benefitting most. A follow-up publication in 2009 failed to show significant effects on inflammatory or $\mathrm{AD}$ biomarkers in (CSF) from a subset of these patients $(\mathrm{n}=35) .^{109}$

Another small, randomized, double-blind, placebocontrolled study from Taiwan was published in 2008 . $^{110}$ The study group included 23 subjects with mild to moderate $\mathrm{AD}$ and 23 subjects with mild cognitive impairment (MCI) randomized to receive either $1.8 \mathrm{~g}$ /day omega-3 PUFA or placebo (olive oil) for 24 weeks of active study participation. Primary outcome measures included the ADAS-cog and the Clinician's Interview-Based Impression of Change scale including caregiver-supplied information (CIBIC-plus). Improvement on the CIBIC-plus was seen for the active treatment versus placebo group overall. ADAS-cog decline did not differ between treatment and placebo groups overall, but a subgroup analysis did demonstrate a beneficial effect of treatment on the ADAS-cog in the MCI group alone. This study again recapitulated the positive effects of omega-3 PUFA supplementation on cognitive decline in early stage memory decline (MCI or early AD) seen in the Freund-Levi study. ${ }^{108}$ No assessment or adjustment for ApoE was performed.

At the 2009 International Conference on Alzheimer's Disease, the preliminary results of two major clinical trials with DHA were presented. The Memory Improvement with DHA Study examined the effects of DHA supplementation (900 mg/day) in a large, multi-center, double-blinded, randomized, placebo-controlled clinical trial with 485 cognitively normal participants, with a mean age of 70 years, over a 6 month treatment period. DHA treatment significantly reduced total errors on the Paired Associates Learning (PAL) subtest of the Cambridge Neuropsychological Test Automated Battery ${ }^{\circledR}$, which was reported as the primary outcome measure. The treated group experienced a doubling of plasma DHA levels and these levels were highly correlated with performance on the PAL. Both compliance and safety were excellent and distributed equally between arms. Data on dietary n-6 intake or ApoE status were not included in the presentation or abstract. The trial was considered positive overall suggesting a benefit of DHA in preventing age-associated cognitive decline. Careful review of the study protocol, however suggests that this primary outcome may have been selected after an interim analysis showed a potential signal, midway during the course of the trial, and that the original outcome measure was the global battery score, which was not significant. These observations cast doubt on the validity of the original conclusions of the study and further demand replication in a future study before the utility of DHA in preventing age associated memory decline can be assessed.

The Alzheimer's Disease Cooperative Study DHA trial examined the effects of DHA ( $2 \mathrm{~g}$ /day) in a large, multi-center, double-blinded, randomized, placebo-controlled clinical trial in 402 subjects with mild to moderate $\mathrm{AD}$, mean MMSE $20.7 \pm 3.6$, and $59 \%$ ApoE $\varepsilon 4$ positive. This trial failed to meet its primary outcome measures of change in ADAS-cog and CDR sum of boxes overall and was considered a negative trial. Treated subjects had elevated levels of both plasma and CSF DHA demonstrating the effectiveness of this regimen in augmenting both peripheral and central DHA stores. Data on dietary n-6 intake were not included in the presentation or abstract. Planned secondary analysis of mild versus moderate stage $\mathrm{AD}$ failed to show a positive effect, in contrast to the previously discussed European trial conducted by Freund-Levi and colleagues. ${ }^{108}$ The planned secondary analysis of the effects of ApoE status on treatment effect demonstrated positive benefit in change on the ADAS-cog and a trend toward a positive effect on the MMSE, but not on the CDR sum of boxes in ApoE $\varepsilon 4$ noncarriers only. These trial results raised speculation on a possible pharmacogenomic interaction between ApoE status and DHA supplementation as has been suggested by others investigating the potential benefits of dietary DHA intake in cross-sectional, epidemiologic, and prospective cohort studies.

The prospective clinical trial data on omega-3 PUFA supplementation to date suggest a potentially positive effect of DHA supplementation on select persons within the study cohorts only. Emerging themes derived from these data include: 1) a potential augmentation of the beneficial effects of DHA in subjects with normal cognition or in the earliest stages of cognitive decline such as MCI or early AD; and 2) a potential role of ApoE status as a modulator of the effect of DHA supplementation in later stage patients. While definitive answers to the potential roles of cognitive status and ApoE genotype influences are lacking, the results of these studies have generated much excitement to further explore 
the potential benefits of omega-3 PUFA supplementation in slowing or preventing cognitive decline.

\section{Ongoing clinical trials of PUFA supplementation}

Several clinical trials investigating the potential cognitive benefits of DHA are underway and can be reviewed in detail on http://clinicaltrials.gov. Ongoing trials are investigating the effects of PUFAs on cognition in a wide range of conditions including in-utero and normal toddler development, pediatric bipolar disorder, autism, post-operative cognitive dysfunction, cardiovascular disease, septic encephalopathy, post traumatic stress disorder, intractable partial seizures, and aging in the frail elderly. Of relevance to the current review, none are focused on assessing a selective response that may be dependent on ApoE status or involve AD directly, although the study of frail aging led by Bruno Vellas at the University Hospital in Toulouse, France could provide further insights into the cognitive decline associated with aging, mild cognitive impairment (MCI), or even early AD depending on how many subjects reach such study endpoints by the end of the 36 month trial period. Additional trials are currently in the planning stages and so further insights into the potential benefits of omega-3 PUFA supplementation in AD may be years away, but nonetheless they are being relentlessly pursued by investigators across the globe that are not dismayed by clinical trial data presented to date.

\section{Conclusion}

Integrating ApoE pharmacogenomics, dietary n-6 intake "pharmacoenvironomics", and cognitive-status/ "pharmaconeuropsychomics" with our understanding of the neuroprotective effects of PUFA supplementation.

Data from the cross-sectional, epidemiologic, and prospective cohort studies of the associations between dietary omega-3 PUFA intake and cognitive status have been largely positive. Dietary habits in these cohorts are likely longstanding rather than short-lived, as in a clinical trial paradigm. These data suggest three emerging themes and possible areas of future exploration: 1) dependence of the potential beneficial effects of omega-3 PUFA on cognitive status, with cognitively normal persons and those in the earliest stages of memory decline (MCI or early AD) showing the greatest benefit; 2) a modulatory effect of dietary n- 6 intake that alters the n-3/n-6 ratio and may negate the positive benefits of $n-3$ supplementation irrespective of $n-3$ intake; and 3) a potential modulatory role of ApoE status that influences the dietary benefits of omega-3 PUFA on cognition. Incomplete assessment of these potential modulators/ confounders across studies prevents firm conclusions from being drawn.

Along these same lines, the prospective clinical trial data on omega-3 PUFA supplementation to date suggest a potentially positive effect of DHA supplementation on select persons within the study cohorts only. Emerging themes derived from these data include: 1) a potential augmentation of the beneficial effects of DHA in subjects with normal cognition or in the earliest stages of cognitive decline such as MCI or early AD; and 2) a potential role of ApoE status as a modulator of the effect of DHA supplementation in later stage patients. While definitive answers to the potential roles of cognitive status and ApoE genotype influences are lacking, the results of these studies have generated considerable interest in the field for future exploration.

The field of pharmacogenomics is emerging as an important contributor to our understanding of responders and non-responders to a variety of therapeutic interventions in many human disease states. The potential modulatory effects of ApoE status on omega-3 PUFA associations with cognitive status may be a prime example of this phenomenon. The effects of lifestyle modifications or confounders that influence the therapeutic efficacy of certain medications are highly appreciated and need to be considered an example of pharmacoenvironomics, with the potential for n-6 intake to completely negate or abrogate the suggested beneficial effects of omega-3 PUFA supplementation on cognitive status. Future studies investigating the association of omega-3 PUFA intake with cognitive status need to take these factors into account, ensuring adequate sampling between ApoE $\varepsilon 4$ carriers and non-carriers or even enriching study populations in non-carriers that may show maximal benefit. FFQ and plasma fatty acid analyses should pay strict attention to n-6 dietary intake in addition to the focus on omega-3 PUFA. Definitive studies in select populations (ie, ApoE e4 non-carriers with low dietary intake of saturated fat and n-6 fatty acids) may assist in the development of rationally-guided, individualized therapies for the subset of AD subjects that may benefit from omega-3 PUFA administration. Such individualized therapy could potentially benefit the $60 \%$ of ApoE $\varepsilon 4$ non-carriers in the global population of more than 35 million persons suffering from this disease (http://www.alz.co.uk/research/files/World\%20Alzheimer \%20Report\%20Executive\%20Summary.pdf), or 21 million affected persons, if omega-3 PUFA supplementation were combined with a regimen of reduced saturated fat and n- 6 fatty acids. The potential influences of ApoE status on 
omega-3 PUFA biology require further exploration and investigation. Linked through their basic roles in lipid biology, ApoE genotype could greatly influence omega-3 PUFA concentrations, transport, metabolism, and sequestration in lipid raft environments essential for healthy neuronal function and synaptic maintenance. Alternative explanations could include combinatorial effects on the production of toxic $\mathrm{A} \beta$ species. While omega-3 PUFA augmentation might decrease the production of $A \beta$, the effects of the ApoE e4 allele could overwhelm or negate such potential benefits of omega-3 PUFA supplementation. Further studies of the basic biology of omega-3 PUFA and ApoE genotype interactions are needed to answer these important questions.

Other considerations include the effect of timing omega-3 PUFA supplementation over the lifespan. Several studies have convincingly demonstrated that omega-3 PUFA supplementation may be more beneficial if administered prior to or in the earliest stages of cognitive decline. It may be that increased omega-3 PUFA levels are protective against cognitive decline and $\mathrm{AD}$, but later play no role in the pathogenic cascade of events that culminate in the clinical and biological expression of fulminate AD, even in the early stages of disease. Alternatively the beneficial effects of omega-3 PUFA augmentation may still exist, but simply be overwhelmed by the strength of the pathological processes responsible for AD once the disease has produced the clinical and biological manifestations of moderate or more severe disease. Lessons learned from the past might suggest a focus on earlier or even preclinical stages of AD as a target for omega-3 PUFA intervention. Such an approach has practical feasibility given the global shift in focus on $\mathrm{AD}$ to the earliest clinical MCI and even preclinical stages of $\mathrm{AD}$ that are being investigated by researchers.

Far from dampening scientific progress in our understanding of the potential benefits of omega-3 PUFA intake, the data obtained from the many studies investigating dietary omega-3 PUFA, while seemingly disparate and even contradictory at times, have resulted in an increased understanding of the potential importance of omega-3 PUFA biology in the CNS and its potential importance in abrogating both age-related cognitive decline and the development of AD. These insights should serve to spur much needed research in this area of neurodegenerative biology and advance our understanding of this devastating disease.

\section{Acknowledgements}

We gratefully acknowledge the many subjects, caregivers, friends, and families of persons with $\mathrm{AD}$ that have engaged in clinical research activities that allow discoveries to be made. We also thank Paula Thomason for critical review and editing of the manuscript.

\section{Disclosures}

Dr Jicha is supported by funding from the NIH/NIA 1 P30 AG028383 and 2R01AG019241, NIH LRP 1 L30 AG032934, and the Sanders-Brown Foundation. Dr Jicha has also received research support for clinical trial activities from NIH/NIA ADCS U01AG010483, Pfizer Inc., Elan Pharmaceuticals, Medivation Inc., and Baxter Inc., and has served as a consultant for Medivation and Martek Inc. in clinical trial development. Dr Markesbery was supported by NIA grants P30 AG028383 (PI), P01 AG05119 (PI), and R01 AG19241 (PI); and by the Healy Family Foundation, and Kleberg Foundation.

\section{In memoriam}

William R Markesbery, MD, internationally known Alzheimer's disease (AD) researcher, died on January 30, 2010, from complications of mycobacterium avium complex at the University of Kentucky (UK) Chandler Medical Center, shortly after co-authoring the present review on omega-3 fatty acids in Alzheimer's disease for the journal, Clinical Interventions in Aging.

Among Dr Markesbery's many achievements, the establishment of the UK Sanders-Brown Center on Aging in 1979 and its continued success over the last 30 years marks his legacy. Soon after the establishment of the Center, Dr Markesbery was awarded one of the first of ten federally funded AD Research Centers (ADCs) with a primary focus on neuropathology. He received 30 years of continuous NIH peer reviewed funding for his research and had over 415 peer reviewed scientific publications. He served as the Chairman of the Medical and Scientific and Advisory Board of the Alzheimer's Association and on the National Advisory Council at NIA. In the early 1980s, Dr Markesbery and collaborators published the first of several studies that disproved the prevailing theory that AD was caused by an accumulation of aluminum and other toxic metals in the brain. Subsequently he and his colleagues were among the first to show that oxidative stress is present early in AD. He later described the neuropathological manifestations in mild cognitive impairment -the earliest detectable clinical phase of AD.

He received numerous awards for his work including the Award for Meritorious Contributions to Neuropathology, the Irving H Shaw Award for Distinguished Service 
in the Field of Health Care, the Medallion for Intellectual Achievement, UK Library Associates, and recognition by the Alltech Lecture Series, University College, Dublin. In 2009 , Dr Markesbery received the prestigious Khachaturian Award from the National Alzheimer's Association for advancing the field of Alzheimer's disease. Bill said "I am grateful to have the opportunity to take part in the investigation of the most devastating disease that affects humanity. It has been a privilege to care for individuals with the disease, support the families and through research, strive to gain a better understanding of Alzheimer's disease so that one day soon we will learn how to prevent the disease in those at risk and stop the progression of those with the disease."

Despite all his accomplishments and his illnesses in the past 15 years, Dr Markesbery remained a tireless researcher and compassionate clinician. He epitomized the meaning of a Southern gentleman and was proud to refer to himself as a 7 th generation Kentuckian. He was admired and respected by all for his humility, kindness, generosity, high standards in all he undertook, and patience. He always had the time to listen to and counsel anyone at any level. He will be greatly missed by family, colleagues, patients and students, but his love of learning, high standards, kindness, and desire to teach will never be forgotten.

\section{References}

1. Jicha GA, Carr SA. Conceptual Evolution in Alzheimer's Disease: Implications for Understanding the Clinical Phenotype of Progressive Neurodegenerative Disease. J Alzheimers Dis. 2010;19(1):253-272.

2. Alzheimer's disease facts and figures. Alzheimers Dement. 2009;5(3):234-270.

3. McKhann G, Drachman D, Folstein M, Katzman R, Price D, Stadlan EM. Clinical diagnosis of Alzheimer's disease: report of the NINCDSADRDA Work Group under the auspices of Department of Health and Human Services Task Force on Alzheimer's Disease. Neurology. 1984;34(7):939-944.

4. Consensus recommendations for the postmortem diagnosis of Alzheimer's disease. The National Institute on Aging, and Reagan Institute Working Group on Diagnostic Criteria for the Neuropathological Assessment of Alzheimer's Disease. Neurobiol Aging. 1997;18 (4 Suppl):S1-S2.

5. Braak H, Braak E. Neuropathological stageing of Alzheimer-related changes. Acta Neuropathol (Berl). 1991;82(4):239-259.

6. Mirra SS, Heyman A, McKeel D, et al. The Consortium to Establish a Registry for Alzheimer's Disease (CERAD). Part II. Standardization of the neuropathologic assessment of Alzheimer's disease. Neurology. 1991;41(4):479-486.

7. Cole GM, Ma QL, Frautschy SA. Omega-3 fatty acids and dementia. Prostaglandins Leukot Essent Fatty Acids. 2009;81(2-3):213-221.

8. Cunnane SC, Plourde M, Pifferi F, Begin M, Feart C, Barberger-Gateau P. Fish, docosahexaenoic acid and Alzheimer's disease. Prog Lipid Res. 2009;48(5):239-256.

9. Boudrault C, Bazinet RP, Ma DW. Experimental models and mechanisms underlying the protective effects of $n-3$ polyunsaturated fatty acids in Alzheimer's disease. J Nutr Biochem. 2009;20(1):1-10.

10. Hooijmans CR, Kiliaan AJ. Fatty acids, lipid metabolism and Alzheimer pathology. Eur J Pharmacol. 2008;585(1):176-196.
11. Markesbery WR, Kryscio RJ, Lovell MA, Morrow JD. Lipid peroxidation is an early event in the brain in amnestic mild cognitive impairment. Ann Neurol. 2005;58(5):730-735.

12. Montine TJ, Morrow JD. Fatty acid oxidation in the pathogenesis of Alzheimer's disease. Am J Pathol. 2005;166(5):1283-1289.

13. Lukiw WJ, Bazan NG. Docosahexaenoic acid and the aging brain. J Nutr. 2008;138(12):2510-2514.

14. Mazza M, Pomponi M, Janiri L, Bria P, Mazza S. Omega-3 fatty acids and antioxidants in neurological and psychiatric diseases: an overview. Prog Neuropsychopharmacol Biol Psychiatry. 2007;31(1):12-26.

15. Wassall SR, Brzustowicz MR, Shaikh SR, Cherezov V, Caffrey M, Stillwell W. Order from disorder, corralling cholesterol with chaotic lipids. The role of polyunsaturated lipids in membrane raft formation. Chem Phys Lipids. 2004;132(1):79-88.

16. Russo GL. Dietary n-6 and n-3 polyunsaturated fatty acids: from biochemistry to clinical implications in cardiovascular prevention. Biochem Pharmacol. 2009;77(6):937-946.

17. Yehuda S, Rabinovitz S, Mostofsky DI. Essential fatty acids and the brain: from infancy to aging. Neurobiol Aging. 2005;26 Suppl 1: 98-102.

18. Gamoh S, Hashimoto M, Sugioka K, et al. Chronic administration of docosahexaenoic acid improves reference memory-related learning ability in young rats. Neuroscience. 1999;93(1):237-241.

19. Kawakita E, Hashimoto M, Shido O. Docosahexaenoic acid promotes neurogenesis in vitro and in vivo. Neuroscience. 2006;139(3): 991-997.

20. Kempermann G, Kuhn HG, Gage FH. More hippocampal neurons in adult mice living in an enriched environment. Nature. 1997;386(6624):493-495.

21. Katakura M, Hashimoto M, Shahdat HM, et al. Docosahexaenoic acid promotes neuronal differentiation by regulating basic helix-loop-helix transcription factors and cell cycle in neural stem cells. Neuroscience. 2009;160(3):651-660.

22. Conklin SM, Gianaros PJ, Brown SM, et al. Long-chain omega-3 fatty acid intake is associated positively with corticolimbic gray matter volume in healthy adults. Neurosci Lett. 2007;421(3):209-212.

23. Barnes J, Scahill RI, Schott JM, Frost C, Rossor MN, Fox NC. Does Alzheimer's disease affect hippocampal asymmetry? Evidence from a cross-sectional and longitudinal volumetric MRI study. Dement Geriatr Cogn Disord. 2005;19(5-6):338-344.

24. Bigler ED, Tate DF, Miller MJ, et al. Dementia, asymmetry of temporal lobe structures, and apolipoprotein E genotype: relationships to cerebral atrophy and neuropsychological impairment. J Int Neuropsychol Soc. 2002;8(7):925-933.

25. Geroldi C, Laakso MP, DeCarli C, et al. Apolipoprotein E genotype and hippocampal asymmetry in Alzheimer's disease: a volumetric MRI study. J Neurol Neurosurg Psychiatry. 2000;68(1):93-96.

26. Likeman M, Anderson VM, Stevens JM, et al. Visual assessment of atrophy on magnetic resonance imaging in the diagnosis of pathologically confirmed young-onset dementias. Arch Neurol. 2005;62(9): 1410-1415.

27. Shi F, Liu B, Zhou Y, Yu C, Jiang T. Hippocampal volume and asymmetry in mild cognitive impairment and Alzheimer's disease: Metaanalyses of MRI studies. Hippocampus. 2009;19(11)1055-1064.

28. Tiemeier H, van Tuijl HR, Hofman A, Kiliaan AJ, Breteler MM. Plasma fatty acid composition and depression are associated in the elderly: the Rotterdam Study. Am J Clin Nutr. 2003;78(1):40-46.

29. Bazan NG. Neuroprotectin D1 (NPD1): a DHA-derived mediator that protects brain and retina against cell injury-induced oxidative stress. Brain Pathol. 2005;15(2):159-166.

30. Lukiw WJ, Cui JG, Marcheselli VL, et al. A role for docosahexaenoic acid-derived neuroprotectin D1 in neural cell survival and Alzheimer disease. J Clin Invest. 2005;115(10):2774-2783.

31. Farooqui AA, Horrocks LA. Phospholipase $\mathrm{A}_{2}$-generated lipid mediators in the brain: the good, the bad, and the ugly. Neuroscientist. 2006;12(3):245-260. 
32. Forlenza OV, Schaeffer EL, Gattaz WF. The role of phospholipase $\mathrm{A}_{2}$ in neuronal homeostasis and memory formation: implications for the pathogenesis of Alzheimer's disease. J Neural Transm. 2007;114(2):231-238.

33. Gattaz WF, Forlenza OV, Talib LL, Barbosa NR, Bottino CM. Platelet phospholipase $\mathrm{A}(2)$ activity in Alzheimer's disease and mild cognitive impairment. J Neural Transm. 2004;111(5):591-601.

34. Schaeffer EL, Forlenza OV, Gattaz WF. Phospholipase $\mathrm{A}_{2}$ activation as a therapeutic approach for cognitive enhancement in early-stage Alzheimer disease. Psychopharmacology (Berl). 2009;202(1-3): $37-51$.

35. Smesny S, Stein S, Willhardt I, Lasch J, Sauer H. Decreased phospholipase $\mathrm{A}_{2}$ activity in cerebrospinal fluid of patients with dementia. J Neural Transm. 2008;115(8):1173-1179.

36. Sun GY, Xu J, Jensen MD, Simonyi A. Phospholipase $A_{2}$ in the central nervous system: implications for neurodegenerative diseases. J Lipid Res. 2004;45(2):205-213.

37. Montine TJ, Montine KS, McMahan W, Markesbery WR, Quinn JF, Morrow JD. F2-isoprostanes in Alzheimer and other neurodegenerative diseases. Antioxid Redox Signal. 2005;7(1-2):269-275.

38. Montine TJ, Beal MF, Cudkowicz ME, et al. Increased CSF F2-isoprostane concentration in probable AD. Neurology. 1999;52(3):562-565.

39. Montine TJ, Markesbery WR, Morrow JD, Roberts LJ 2nd. Cerebrospinal fluid F2-isoprostane levels are increased in Alzheimer's disease. Ann Neurol. 1998;44(3):410-413.

40. Nourooz-Zadeh J, Liu EH, Yhlen B, Anggard EE, Halliwell B. F4-isoprostanes as specific marker of docosahexaenoic acid peroxidation in Alzheimer's disease. J Neurochem. 1999;72(2):734-740.

41. Pratico D, V MYL, Trojanowski JQ, Rokach J, Fitzgerald GA. Increased F2-isoprostanes in Alzheimer's disease: evidence for enhanced lipid peroxidation in vivo. FASEB J. 1998;12(15):1777-1783.

42. Reich EE, Markesbery WR, Roberts LJ 2nd, Swift LL, Morrow JD, Montine TJ. Quantification of F-ring and D-/E-ring isoprostanes and neuroprostanes in Alzheimer's disease. Adv Exp Med Biol. 2001;500:253-256.

43. Reich EE, Markesbery WR, Roberts LJ 2nd, Swift LL, Morrow JD, Montine TJ. Brain regional quantification of F-ring and D-/E-ring isoprostanes and neuroprostanes in Alzheimer's disease. Am J Pathol. 2001;158(1):293-297.

44. Hardy J. Has the amyloid cascade hypothesis for Alzheimer's disease been proved? Curr Alzheimer Res. 2006;3(1):71-73.

45. Hardy JA, Higgins GA. Alzheimer's disease: the amyloid cascade hypothesis. Science. 1992;256(5054):184-185.

46. Selkoe DJ. Soluble oligomers of the amyloid beta-protein impair synaptic plasticity and behavior. Behav Brain Res. 2008;192(1):106-113.

47. Cole SL, Vassar R. BACE1 structure and function in health and Alzheimer's disease. Curr Alzheimer Res. 2008;5(2):100-120.

48. Stockley JH, O'Neill C. The proteins BACE1 and BACE2 and betasecretase activity in normal and Alzheimer's disease brain. Biochem Soc Trans. 2007;35(Pt 3):574-576.

49. Stockley JH, O'Neill C. Understanding BACE1: essential protease for amyloid-beta production in Alzheimer's disease. Cell Mol Life Sci. 2008;65(20):3265-3289.

50. Stockley JH, Ravid R, O'Neill C. Altered beta-secretase enzyme kinetics and levels of both BACE1 and BACE2 in the Alzheimer's disease brain. FEBS Lett. 2006;580(28-29):6550-6560.

51. Willem M, Lammich S, Haass C. Function, regulation and therapeutic properties of beta-secretase (BACE1). Semin Cell Dev Biol. 2009;20(2):175-182.

52. Hashimoto M, Shahdat HM, Yamashita S, et al. Docosahexaenoic acid disrupts in vitro amyloid beta(1-40) fibrillation and concomitantly inhibits amyloid levels in cerebral cortex of Alzheimer's disease model rats. J Neurochem. 2008;107(6):1634-1646.

53. Hossain S, Hashimoto M, Katakura M, Miwa K, Shimada T, Shido O. Mechanism of docosahexaenoic acid-induced inhibition of in vitro Abeta1-42 fibrillation and Abeta1-42-induced toxicity in SH-S5Y5 cells. J Neurochem. 2009;111(2):568-579.
54. Ehehalt R, Keller P, Haass C, Thiele C, Simons K. Amyloidogenic processing of the Alzheimer beta-amyloid precursor protein depends on lipid rafts. J Cell Biol. 2003;160(1):113-123.

55. Green KN, Martinez-Coria H, Khashwji H, et al. Dietary docosahexaenoic acid and docosapentaenoic acid ameliorate amyloid-beta and tau pathology via a mechanism involving presenilin 1 levels. $J$ Neurosci. 2007;27(16):4385-4395.

56. Oksman M, Iivonen H, Hogyes E, et al. Impact of different saturated fatty acid, polyunsaturated fatty acid and cholesterol containing diets on beta-amyloid accumulation in APP/PS1 transgenic mice. Neurobiol Dis. 2006;23(3):563-572.

57. Riddell DR, Christie G, Hussain I, Dingwall C. Compartmentalization of beta-secretase (Asp2) into low-buoyant density, noncaveolar lipid rafts. Curr Biol. 2001;11(16):1288-1293.

58. Calon F, Lim GP, Yang F, et al. Docosahexaenoic acid protects from dendritic pathology in an Alzheimer's disease mouse model. Neuron. 2004;43(5):633-645.

59. Lim GP, Calon F, Morihara T, et al. A diet enriched with the omega-3 fatty acid docosahexaenoic acid reduces amyloid burden in an aged Alzheimer mouse model. J Neurosci. 2005;25(12):3032-3040.

60. Hashimoto M, Hossain S, Agdul H, Shido O. Docosahexaenoic acidinduced amelioration on impairment of memory learning in amyloid beta-infused rats relates to the decreases of amyloid beta and cholesterol levels in detergent-insoluble membrane fractions. Biochim Biophys Acta. 2005;1738(1-3):91-98.

61. Hashimoto M, Hossain S, Shimada T, et al. Docosahexaenoic acid provides protection from impairment of learning ability in Alzheimer's disease model rats. J Neurochem. 2002;81(5):1084-1091.

62. Hashimoto M, Tanabe Y, Fujii Y, Kikuta T, Shibata H, Shido O. Chronic administration of docosahexaenoic acid ameliorates the impairment of spatial cognition learning ability in amyloid beta-infused rats. $J$ Nutr. 2005;135(3):549-555.

63. Hashimoto M, Hossain S, Shimada T, Shido O. Docosahexaenoic acid-induced protective effect against impaired learning in amyloid beta-infused rats is associated with increased synaptosomal membrane fluidity. Clin Exp Pharmacol Physiol. 2006;33(10):934-939.

64. de la Torre JC. Alzheimer's disease is a vasocognopathy: a new term to describe its nature. Neurol Res. 2004;26(5):517-524.

65. Engelberg H. Pathogenic factors in vascular dementia and Alzheimer's disease. Multiple actions of heparin that probably are beneficial. Dement Geriatr Cogn Disord. 2004;18(3-4):278-298.

66. Weller RO, Cohen NR, Nicoll JA. Cerebrovascular disease and the pathophysiology of Alzheimer's disease. Implications for therapy. Panminerva Med. 2004;46(4):239-251.

67. Yip AG, McKee AC, Green RC, et al. APOE, vascular pathology, and the AD brain. Neurology. 2005;65(2):259-265.

68. Zekry D, Hauw JJ, Gold G. Mixed dementia: epidemiology, diagnosis, and treatment. J Am Geriatr Soc. 2002 Aug;50(8):1431-1438.

69. Hornstra G. Influence of dietary fat type on arterial thrombosis tendency. J Nutr Health Aging. 2001;5(3):160-166.

70. Dimitrow PP, Jawien M. Pleiotropic, cardioprotective effects of omega-3 polyunsaturated fatty acids. Mini Rev Med Chem. 2009;9(9): $1030-1039$

71. He K. Fish, long-chain omega-3 polyunsaturated fatty acids and prevention of cardiovascular disease--eat fish or take fish oil supplement? Prog Cardiovasc Dis. 2009;52(2):95-114.

72. Lavie CJ, Milani RV, Mehra MR, Ventura HO. Omega-3 polyunsaturated fatty acids and cardiovascular diseases. $\mathrm{J} \mathrm{Am} \mathrm{Coll} \mathrm{Cardiol.}$ 2009;54(7):585-594.

73. Marik PE, Varon J. Omega-3 dietary supplements and the risk of cardiovascular events: a systematic review. Clin Cardiol. 2009;32(7): 365-372.

74. Mozaffarian D. Fish, n-3 fatty acids, and cardiovascular haemodynamics. J Cardiovasc Med (Hagerstown). 2007;8 Supp1 1:S23-S26.

75. Mozaffarian D. Fish, mercury, selenium and cardiovascular risk: current evidence and unanswered questions. Int J Environ Res Public Health. 2009;6(6):1894-1916. 
76. Willett WC. The role of dietary n-6 fatty acids in the prevention of cardiovascular disease. J Cardiovasc Med (Hagerstown). 2007;8 Suppl 1: S42-S45.

77. Marzolo MP, Bu G. Lipoprotein receptors and cholesterol in APP trafficking and proteolytic processing, implications for Alzheimer's disease. Semin Cell Dev Biol. 2009;20(2):191-200.

78. Stefani M, Liguri G. Cholesterol in Alzheimer's disease: unresolved questions. Curr Alzheimer Res. 2009;6(1):15-29.

79. $\mathrm{Bu}$ G. Apolipoprotein E and its receptors in Alzheimer's disease: pathways, pathogenesis and therapy. Nat Rev Neurosci. 2009;10(5): 333-344.

80. de Chaves EP, Narayanaswami V. Apolipoprotein E and cholesterol in aging and disease in the brain. Future Lipidol. 2008;3(5): 505-530.

81. Kim J, Basak JM, Holtzman DM. The role of apolipoprotein E in Alzheimer's disease. Neuron. 2009;63(3):287-303.

82. Schipper HM. Apolipoprotein E: Implications for AD neurobiology, epidemiology and risk assessment. Neurobiol Aging. 292009.

83. Beach TG, Wilson JR, Sue LI, et al. Circle of Willis atherosclerosis: association with Alzheimer's disease, neuritic plaques and neurofibrillary tangles. Acta Neuropathol. 2007;113(1):13-21.

84. Honig LS, Kukull W, Mayeux R. Atherosclerosis and AD: analysis of data from the US National Alzheimer's Coordinating Center. Neurology. 2005;64(3):494-500.

85. Roher AE, Esh C, Kokjohn TA, et al. Circle of willis atherosclerosis is a risk factor for sporadic Alzheimer's disease. Arterioscler Thromb Vasc Biol. 2003;23(11):2055-2062.

86. Roher AE, Esh C, Rahman A, Kokjohn TA, Beach TG. Atherosclerosis of cerebral arteries in Alzheimer disease. Stroke. 2004 Nov;35(11 Suppl 1): 2623-2627.

87. Castano EM, Prelli F, Wisniewski T, et al. Fibrillogenesis in Alzheimer's disease of amyloid beta peptides and apolipoprotein E. Biochem J. 1995;306(Pt 2):599-604.

88. Soto C, Castano EM, Prelli F, Kumar RA, Baumann M. Apolipoprotein $\mathrm{E}$ increases the fibrillogenic potential of synthetic peptides derived from Alzheimer's, gelsolin and AA amyloids. FEBS Lett. 1995;371(2):110-114.

89. Kalmijn S, Launer LJ, Ott A, Witteman JC, Hofman A, Breteler MM. Dietary fat intake and the risk of incident dementia in the Rotterdam Study. Ann Neurol. 1997;42(5):776-782.

90. Engelhart MJ, Geerlings MI, Ruitenberg A, et al. Diet and risk of dementia: Does fat matter?: The Rotterdam Study. Neurology. 2002;59(12):1915-1921.

91. Conquer JA, Tierney MC, Zecevic J, Bettger WJ, Fisher RH. Fatty acid analysis of blood plasma of patients with Alzheimer's disease, other types of dementia, and cognitive impairment. Lipids. 2000;35(12):1305-1312.

92. Laurin D, Verreault R, Lindsay J, Dewailly E, Holub BJ. Omega-3 fatty acids and risk of cognitive impairment and dementia. JAlzheimers Dis. 2003;5(4):315-322.

93. Kroger E, Verreault R, Carmichael PH, et al. Omega-3 fatty acids and risk of dementia: the Canadian Study of Health and Aging. Am J Clin Nutr. 2009;90(1):184-192.

94. Barberger-Gateau P, Letenneur L, Deschamps V, Peres K, Dartigues JF, Renaud S. Fish, meat, and risk of dementia: cohort study. BMJ. 2002;325(7370):932-933.
95. Barberger-Gateau P, Raffaitin C, Letenneur L, et al. Dietary patterns and risk of dementia: the Three-City cohort study. Neurology. 2007;69(20):1921-1930.

96. Otsuka M, Yamaguchi K, Ueki A. Similarities and differences between Alzheimer's disease and vascular dementia from the viewpoint of nutrition. Ann N Y Acad Sci. 2002;977:155-161.

97. Tully AM, Roche HM, Doyle R, et al. Low serum cholesteryl esterdocosahexaenoic acid levels in Alzheimer's disease: a case-control study. Br J Nutr. 2003;89(4):483-489.

98. Morris MC, Evans DA, Bienias JL, et al. Consumption of fish and n-3 fatty acids and risk of incident Alzheimer disease. Arch Neurol. 2003;60(7):940-946.

99. Morris MC, Evans DA, Tangney CC, Bienias JL, Wilson RS. Fish consumption and cognitive decline with age in a large community study. Arch Neurol. 2005;62(12):1849-1853.

100. Huang TL, Zandi PP, Tucker KL, et al. Benefits of fatty fish on dementia risk are stronger for those without APOE epsilon4. Neurology. 2005;65(9):1409-1414.

101. Schaefer EJ, Bongard V, Beiser AS, et al. Plasma phosphatidylcholine docosahexaenoic acid content and risk of dementia and Alzheimer disease: the Framingham Heart Study. Arch Neurol. 2006;63(11): 1545-1550.

102. Beydoun MA, Kaufman JS, Satia JA, Rosamond W, Folsom AR. Plasma n-3 fatty acids and the risk of cognitive decline in older adults: the Atherosclerosis Risk in Communities Study. Am J Clin Nutr. 2007;85(4):1103-1111.

103. van Gelder BM, Tijhuis M, Kalmijn S, Kromhout D. Fish consumption, n-3 fatty acids, and subsequent 5-y cognitive decline in elderly men the Zutphen Elderly Study. Am J Clin Nutr. 2007;85(4):1142-1147.

104. Dangour AD, Allen E, Elbourne D, Fletcher A, Richards M, Uauy R. Fish consumption and cognitive function among older people in the UK: baseline data from the OPAL study. $J$ Nutr Health Aging. 2009;13(3):198-202.

105. Whalley LJ, Deary IJ, Starr JM, et al. n-3 Fatty acid erythrocyte membrane content, APOE varepsilon4, and cognitive variation: an observational follow-up study in late adulthood. Am J Clin Nutr. 2008;87(2):449-454.

106. Boston PF, Bennett A, Horrobin DF, Bennett CN. Ethyl-EPA in Alzheimer's disease - a pilot study. Prostaglandins Leukot Essent Fatty Acids. 2004;71(5):341-346.

107. Kotani S, Sakaguchi E, Warashina S, et al. Dietary supplementation of arachidonic and docosahexaenoic acids improves cognitive dysfunction. Neurosci Res. 2006;56(2):159-164.

108. Freund-Levi Y, Eriksdotter-Jonhagen M, Cederholm T, et al. Omega-3 fatty acid treatment in 174 patients with mild to moderate Alzheimer disease: OmegAD study: a randomized double-blind trial. Arch Neurol. 2006;63(10):1402-1408.

109. Freund-Levi Y, Hjorth E, Lindberg C, et al. Effects of omega-3 fatty acids on inflammatory markers in cerebrospinal fluid and plasma in Alzheimer's disease: the OmegAD study. Dement Geriatr Cogn Disord. 2009;27(5):481-490.

110. Chiu CC, Su KP, Cheng TC, et al. The effects of omega-3 fatty acids monotherapy in Alzheimer's disease and mild cognitive impairment: a preliminary randomized double-blind placebo-controlled study. Prog Neuropsychopharmacol Biol Psychiatry. 2008;32(6):1538-1544.
Clinical Interventions in Aging

\section{Publish your work in this journal}

Clinical Interventions in Aging is an international, peer-reviewed journal focusing on evidence-based reports on the value or lack thereof of treatments intended to prevent or delay the onset of maladaptive correlates of aging in human beings. This journal is indexed on PubMed Central, MedLine, the American Chemical Society's 'Chemical

\section{Dovepress}

Abstracts Service' (CAS), Scopus and the Elsevier Bibliographic databases. The manuscript management system is completely online and includes a very quick and fair peer-review system, which is all easy to use. Visit http://www.dovepress.com/testimonials.php to read real quotes from published authors. 